Brazilian Journal of Forensic Sciences, Medical Law and Bioethics

\title{
La Hermenéutica-Dialéctica como Método de Análisis Cualitativo en Enfermería
}

\section{The Hermeneutic-Dialectical as a Qualitative Research Analysis in Nursing}

\author{
A Hermenêutica-Dialética como Método de Análise Qualitativa em Enfermagem \\ Víctor Federico Rodríguez Nava ${ }^{1, *}$, Carla Aparecida Arena Ventura ${ }^{2}$, \\ Leticia Casique Casique ${ }^{1}$, Alejandra Hernández Castañón ${ }^{3}$ \\ ${ }^{1}$ División de Ciencias de la Salud e Ingenierías, Campus Celaya-Salvatierra, \\ Universidad Autónoma de Guanajuato, Guanajuato, México \\ ${ }^{2}$ Escola de Enfermagem de Ribeirão Preto, Departamento de Enfermería Psiquiátrica y Ciencias \\ Humanas, Universidad de São Paulo, Brasil \\ ${ }^{3}$ Facultad de Ciencias Políticas y Sociales. Universidad Autónoma de Querétaro, Querétaro, México \\ *Corresponding author. E-mail: victorrguez7@hotmail.com
}

Resumen. El análisis hermenéutico-dialéctico, es un método que de manera consciente o inconsciente el investigador en enfermería emplea durante el proceso de análisis cualitativo de datos. Este método de análisis, surge como una propuesta de Jürgen Habermas en su diálogo con Gadamer, a fin de mostrarle que la universalidad de la hermenéutica está en la razón cuando el sujeto es capaz de hacer una crítica a la tradición y la autoridad con vistas de transformar la realidad. De manera aislada, el concepto de hermenéutica ha sufrido una metamorfosis semántica en el acontecer histórico, puesto que paso de ser un arte o teoría para explicar, traducir o interpretar textos bíblicos, filológicos y jurídicos, hasta llegar a convertirse en una corriente filosófica que se caracteriza por la idea que: "La verdad es fruto de una interpretación". Por su parte, la dialéctica es considerada como el arte de la conversación, el diálogo, la diferencia, la oposición, el disenso, la polémica o la argumentación. Es una rama de la filosofía que evidencia la contradicción que se da entre dos tesis, generando a partir de ellas un producto final denominado proceso resultante. Por lo anterior, Habermas, al considerar las ventajas y desventajas de ambos métodos, menciona que su propuesta de análisis Hermenéutico-Dialéctico, busca la interpretación de la hermenéutica con el ejercicio crítico de la dialéctica, situación que dentro del análisis cualitativo de datos en enfermería, puede ser empleada para descubrir semejanzas, 
contradicciones, así como ser interpretativo y critico al generar conocimiento dentro del gremio de enfermería.

Palabras clave: Hermenéutica; Dialéctica; Investigación en enfermería.

Abstract. The hermeneutic-dialectical analysis is a method that a nursing researcher uses consciously or unconsciously during the process of qualitative data analysis. This method begins as a proposal by Jürgen Habermas in his dialogue with Gadamer in order to show him that the universality of hermeneutics is in reason when the subject is capable of criticizing tradition and authority with an overview to transform reality. In isolation, the concept of hermeneutics has suffered a semantic metamorphosis in historical events; from being an art or theory in explaining, translating or interpreting biblical, philosophical and legal texts, until it becomes a philosophical school characterized by the idea: "Truth is the fruit of interpretation. Alternatively, dialectic considers the art of conversation, dialogue, difference, opposition, discrepancy, controversy or argumentation. Dialectic is a branch of philosophy that confirms conflict between two judgments, generating from these a final product named the resulting process. Because of this, when considering the advantages and disadvantages of both methods, Habermas remarks that his proposal of Hermeneutic-Dialectic analysis seeks the interpretation of hermeneutics with the critical application of dialectic, that within the qualitative data analysis in nursing, can be used to discover resemblances, inconsistencies, as well as being interpretative and critical in generating knowledge inside the nursing profession.

Keywords: Hermeneutics; Dialectic; Nursing research.

Resumo. A análise hermenêutica-dialética é um método que o pesquisador em enfermagem usa conscientemente ou inconscientemente durante o processo de análise de dados qualitativos. Este método de análise emerge como uma proposta de Jürgen Habermas em seu diálogo com Gadamer, para mostrar-Ihe que a universalidade da hermenêutica é na razão em que o sujeito é capaz de fazer uma crítica da tradição e autoridade em vista de transformar a realidade. De forma isolada, o conceito de hermenêutica sofreu uma metamorfose semântica em eventos históricos, já que passou de ser uma arte ou uma teoria para explicar, traduzir ou interpretar textos bíblicos, filológicos e legais, até se tornar uma corrente filosófica que se caracteriza pela idéia de que: "A verdade é o resultado de uma interpretação". Por sua vez, a dialética é considerada a arte da conversa, do diálogo, da diferença, da oposição, da dissidência, da controvérsia ou da argumentação. É um ramo da filosofia que demonstra a contradição que ocorre entre duas teses, gerando um produto final chamado processo resultante. Portanto, Habermas, considerando as vantagens e desvantagens de ambos os métodos, menciona que sua proposta de análise hismenêutica- 
dialética busca a interpretação da hermenêutica com o exercício crítico da dialética, situação que, dentro da análise de dados qualitativos em enfermagem, pode ser usada para descobrir semelhanças, contradições, além de ser interpretativa e crítica ao gerar conhecimento na profissão de enfermagem.

Palavras-chave: Hermenêutica; Dialétic; Pesquisa em enfermagem.

\section{Introducción}

Enfermería como ciencia, tiene la obligación ética y moral de ampliar, utilizar y consultar la mejor evidencia científica disponible con el objetivo de sustentar los cuidados proporcionados ${ }^{1}$, situaciones que pueden llevarse a cabo a través del acercamiento a la verdad a través de los paradigmas cuantitativo y cualitativo que ofrece la ciencia para generar investigación básica o aplicada $^{2}$. En este orden de ideas, la investigación cualitativa cuenta con varios métodos o aproximaciones para el análisis de datos y uno de ellos es el método hermenéutico-dialéctico ${ }^{3}$. Este método de análisis, fue inicialmente descrito por Jürgen Habermas en el diálogo establecido con Gadamer en 1987 con el objetivo de sintetizar los procesos comprensivos de la hermenéutica y críticos de la dialéctica logrando de esta manera, que las limitaciones de la hermenéutica sean compensadas por la dialéctica y las limitaciones de la dialéctica sean apoyadas por la Hermenéutica, permitiendo en todo momento del análisis ser comprensivo, crítico, y descubrir las semejanzas y contradicciones al interpretar la realidad social ${ }^{4}$.

Por lo anterior, es que es necesario comprender la evolución epistemológica de cada uno de los métodos antes mencionados hasta llegar a la conjunción necesaria de los mismos, con la intención de comprender que la articulación Hermenéutica-Dialéctica surge como un método que permite interpretar y argumentar los fenómenos identificados sin fragmentación y puede ser empleado en el análisis de datos cualitativos en enfermería.

\section{Hermenéutica}

De acuerdo con Calixto Morales y Franca D'Agostini, el concepto de hermenéutica ha sufrido una metamorfosis semántica en el acontecer histórico, puesto que paso de ser un arte o teoría para explicar, traducir o interpretar textos bíblicos, filológicos y jurídicos, hasta llegar a convertirse en nuestros días como una corriente filosófica contemporánea que se caracteriza por la idea de que "la verdad es fruto de una 
interpretación"5,6. Desde el punto de vista etimológico, el término hermenéutica proviene del verbo griego Hermeneuein, el cual está ligado a las funciones del dios griego Hermes, hijo de Zeus y Maya, quien era el encargado en el olimpo de transmitir e interpretar a los hombres mensajes y órdenes divinas para que estas fueran claramente comprendidas y acatadas ${ }^{7}$.

Por esta razón, en un inicio no se habla simplemente de Hermenéutica, si no de diversos tipos de hermenéuticas específicas a saber: Hermenéutica teológica: Se encargaba de la interpretación de textos sagrados y todo aquello relacionado con la divinidad, lo cual le da a este tipo de Hermenéutica, un sentido o significado salvífico a la escritura sagrada como un texto absoluto ${ }^{8}$, Hermenéutica Filológica: Determinaba el sentido autentico de los escritos antiguos, esclareciendo las condiciones de una interpretación válidamente universal, abandonando la idea de adquisición de la comprensión por otorgamiento de un don divino ${ }^{9}$, Hermenéutica jurídica: Rama de la ciencia del derecho que trata de la correcta interpretación de las normas que lo constituyen, con el objetivo de facilitar la labor del juez al dar soporte a sus decisiones al aplicar las leyes ${ }^{9,10}$ y la Hermenéutica filosófica: En palabras de Gadamer, este tipo de Hermenéutica es considerada como el arte del entendimiento y consiste en reconocer como principio supremo el dejar abierto el diálogo, comprender la realidad social y considerar en todo momento el contexto socio histórico en que sucede el hecho ${ }^{11,12}$.

De acuerdo con George Reyes y Luis Enrique de Santiago respectivamente, Friedrich Schleiermacher es considerado como el padre de la hermenéutica moderna y el máximo exponente de la hermenéutica romántica ${ }^{13,14}$. Schleiermacher criticaba la hermenéutica por su falta de unidad debido a que ésta no existía como un campo general, sino como una pluralidad de hermenéuticas especializadas ${ }^{15}$. Sin embargo, su hermenéutica puede considerarse como una dimensión fundamental de la filosofía y menciona que como "Arte de comprender", la hermenéutica no existe como una materia general, sino como una pluralidad de hermenéuticas especiales"14. Para Schleiermacher en esa época, la tarea de la hermenéutica era: comprender en la lengua y comprender en la persona que habla ${ }^{16}$ "Procurando entender el discurso tan bien como el autor y después mejor que ér17.

Schleiermacher propuso un sistema circular al que ahora conocemos como círculo hermenéutico a través del cual menciona algunas condiciones para su correcta comprensión: a) El todo se compone de partes, las cuales deben ser 
comprendidas antes de contemplar la totalidad; b) Cada parte representa ella misma el todo, todo que se descompone a su vez en partes individuales ${ }^{14}$. Por tanto, el contexto se explica en función de sus partes y las relaciones existentes entre las mismas, la palabra dentro de la frase, la frase dentro del capítulo, el capítulo dentro de todo el texto, el texto inscrito en su tiempo y viceversa ${ }^{18}$.

Inspirado en Scheleiermarcher, Wilhelm Dilthey menciona que la hermenéutica es la interpretación general de las manifestaciones del espíritu expresadas en signos y alusivas a las vivencias, en donde el fundamento metódico de esta hermenéutica no es la "Explicación", sino la "Comprensión", debido a que la naturaleza se explica, pero la vida espiritual se "Comprende" comprensión al "Proceso en el cual se llega a conocer la vida psíquica partiendo de sus manifestaciones sensiblemente dadas", es decir: aprendemos lo psíquico a través de múltiples manifestaciones (Signos, gestos, inscripciones) ${ }^{20}$. Es así que para Dilthey en el texto se expresan los sentimientos del autor y los intérpretes deben intentar ponerse en el lugar del autor para revivir el acto creado ${ }^{19}$.

Dilthey en su obra "Origen de la Hermenéutica", da un paso importante y definitivo más allá de lo presentado por Schleiermacher debido a que sostiene: "No sólo los textos escritos, son objeto natural de interpretación hermenéutica, sino que toda expresión de vida humana puede llegar a ser interpretada hermenéuticamente"21.

Por otro lado, para Martin Heidegger la hermenéutica no es un método que se puede diseñar, enseñar y aplicar por los investigadores ${ }^{22}$. Él menciona que el ser humano es un ser interpretativo, por tanto, la interpretación no es un instrumento para adquirir conocimiento, debido a que es el modo natural de los seres humanos ${ }^{15}$. Heidegger, al igual que los anteriores filósofos, menciona que no existe una verdad pura, ya que estamos influidos por nuestra condición de seres históricos, con nuestro modo de ver; con nuestras actitudes y conceptos ligados a la lengua; con valores, normas culturales y estilos de pensamiento y de vida ${ }^{21}$, por lo tanto menciona, que es imposible comprender los fenómenos con una mente pura y sin preconceptos históricamente construidos. Todo esto aproxima al investigador a cualquier expresión de la vida humana, con expectativas y prejuicios de lo que pudiera ser el objeto observado ${ }^{15}$.

Para Heidegger, la interpretación es un desarrollo posterior a la comprensión, es así que la interpretación se apropia de la comprensión y se mueve 
en un círculo de manera constante, provocando que algo sea expreso o explicito ${ }^{23}$, por lo cual es enfático en mencionar que: "La Primera, constante y última tarea de la interpretación consiste en no dejar que la experiencia previa y la anticipación sean dados por simples ocurrencias y opiniones populares", sino en asegurarse del carácter científico del tema mediante la elaboración de esa estructura de prioridad a partir de las cosas mismas" 24 .

El alemán Hans-Georg Gadamer, quien es considerado como el impulsor de la filosofía hermenéutica contemporánea y quien recogió los hallazgos de Schleiermacher, Wilhelm Dilthey y Martin Heidegger, menciona que la hermenéutica filosófica es la teoría de la verdad y a la vez un arte de interpretación ${ }^{12}$. Considera que nunca será posible tener un conocimiento objetivo del significado de un texto o cualquier otra expresión de la vida psíquica, ya que estamos influidos por nuestra condición de seres históricos con nuestro modo de ver, nuestras actitudes, nuestros conceptos ligados a la lengua, con valores, normas culturales y estilos de pensamiento propios ${ }^{21}$.

Para Gadamer comprender es ejercer la capacidad de colocarse a sí mismo en el lugar del otro: el "tú" del pasado o del presente, con el cual yo, conformo la misma humanidad ${ }^{25}$. En este sentido, la hermenéutica es necesaria cuando desaparece la comprensión, es decir: "Cuando no hay un acuerdo o consenso" entre el texto y el intérprete ${ }^{26}$.

En su hermenéutica, Gadamer retoma el círculo hermenéutico propuesto por Schleiermacher y menciona que siempre hay preconcepciones, es decir: concebimos un posible contenido antes de leer un texto, pero al ingresar al círculo la interpretación final se basa en preconcepciones que van siendo corregidas a medida que se comprueba su falta de adecuación a las cosas mismas ${ }^{24}$, por lo cual podemos deducir que la utilidad del circulo hermenéutico que es infinito, es obtener una retroalimentación del conocimiento, contenido o información que se esté trabajando, para llegar a una interpretación más detallada y global, dado que no existe la verdad, si no que el hermeneuta dice su verdad ${ }^{27}$.

Gadamer, siguiendo a Heidegger, muestra que nuestra comprensión está situada y tiene una fusión de horizontes (una interacción dialéctica entre las expectativas del intérprete y el significado del texto o acto humano ${ }^{28}$ una vez que no somos una conciencia ante un mundo objetivo por conocer, sino que estamos siempre en una situación histórica, concreta y lingüística desde la cual 
interpretamos ${ }^{29}$. Además menciona que: "El ser que puede ser comprendido es lenguaje"30. Sin embargo, con tal afirmación Gadamer no está diciendo que todo el ser puede comprenderse a través del lenguaje, sino que nuestra relación con el mundo es lingüística, es la palabra la que permite comprender, pero lo comprendido también es lenguaje ${ }^{29}$, debido a que todo el mundo humano es lenguaje porque todo está ya significado y comprendido como algo ${ }^{31}$.

Para concluir, Gadamer sugiere que al interpretar, se procure entender al autor mejor de lo que el mismo lo hubiera hecho, intentando develar lo que quedó inconsciente $u$ obscuro para él ${ }^{25}$.

\section{Dialéctica}

Por otra parte, la palabra dialéctica procede del griego (dialektiké), que significa: Arte de la conversación, el diálogo, la diferencia, la oposición, el disenso, la polémica o la discusión $n^{32,33}$. Ha recibido en el correr de los años distintos significados, estando concebida en primer instancia como el arte del discurso, hasta ser considerada para designar las enseñanzas dirigidas al aprendizaje de la discusión ${ }^{33,34}$.

Los inicios de la dialéctica nos remontan a la antigua Grecia en la que Sócrates con el método Mayéutico y la ironía socrática empleaba el diálogo y con ello hacia ver a su interlocutor que aquello que creía no saber en el fondo sí lo sabía ${ }^{35}$. Es así que mediante la dialéctica (diálogo), el propio individuo desarrollaba conceptos a partir de sus respuestas. Por lo anterior, para Sócrates la dialéctica es un método de conocimiento que busca la verdad a partir de hábiles preguntas hasta el punto de orillar al interlocutor a reconocer su ignorancia y el desconocimiento de la definición que se está buscando ${ }^{36}$ y está constituida por dos elementos estrechamente unidos, a) saber preguntar y b) saber responder ${ }^{37}$. Sócrates mencionaba que no aprendemos del maestro y que más bien es en el interior de cada persona en donde se encuentra la respuesta a nuestras interrogantes ${ }^{38}$.

Para Platón, la dialéctica es el arte del discurso o la discusión ${ }^{39}$, el consideraba que la dialéctica es la actitud propia del verdadero filósofo, de aquel que trata de llegar a la verdad por medio del diálogo tal y como lo pensaba Sócrates) ${ }^{40}$. Es una técnica que busca la cooperación con el fin último de alcanzar la verdad ${ }^{41}$.

Para Platón la dialéctica (Discurso), permite contraponer argumentaciones aparentemente opuestas y frecuentemente complementarias, para posibilitar un ascenso a la verdad mediante la explicación de tales argumentaciones ${ }^{40}$. La 
dialéctica de Platón está conformada por dos momentos a) La Synagogé, del griego composición o unificación, el cual consiste en la unión y conjunción de varias características a una única idea que las englobe, b) La Diáiresis, del griego separación, división o particularización, que corresponde a un proceso por el cual Platón efectúa la división de una idea por géneros y especies para llegar a lo particular conforme a una definición común ${ }^{37,41,42}$. Por lo tanto, podemos deducir que la dialéctica en Platón no se basa en la opinión, sino en la verdad, con fines de alcanzar una definición satisfactoria ${ }^{41}$.

En Aristóteles la dialéctica es el arte de descubrir lo oculto, lo cual constituye un discurso a través del camino ${ }^{43}$, es una lógica de lo probable, una técnica que ayuda a discutir con habilidad, en ella se conocen opiniones o ideas comunes, reconocidas y aceptadas mayoritariamente en la sociedad, que pueden ser verdaderas o falsas, pero que se caracterizan por el hecho de haber sido aceptadas de un modo generalizado y que son usadas a la hora de "combatir" y "vencer" las tesis del interlocutor ${ }^{37}$.

Por su parte, los estoicos relacionaron la dialéctica con la lógica, para ellos esta última estaba compuesta por dos elementos: la retórica, considerada como la ciencia del bien hablar, la buena expresión y la propia dialéctica, (ciencia de la recta discusión en los discursos por preguntas y respuestas) ${ }^{44}$. Es así que la dialéctica para los estoicos es considerada como la ciencia de lo verdadero y de lo falso, de lo que no es ni verdadero ni falso ${ }^{37,45}$. En este sentido, Kant desarrolló la antinomia, misma que designa un conflicto entre dos ideas, oposiciones o actitudes, un conflicto entre leyes producida por la naturaleza especulativa de la razón, mismas que son entendidas como tesis y antítesis, donde la primera de ella afirma el punto de vista de quien defiende para poder explicar lo que ocurre en el mundo y la antítesis a su vez, representa la posición determinista o naturalista ${ }^{46}$.

Durante el romanticismo Alemán, Hegel menciono que la dialéctica es una metodología filosófica para buscar la verdad a partir de proposiciones aparentemente contradictorias ${ }^{47}$. Según Hegel, una cosa es ella misma y su contrario, por lo cual menciona que para él, la Dialéctica es la propia naturaleza del pensamiento, puesto que es la reconciliación de dos afirmaciones opuestas ${ }^{48}$.

Debido a lo anterior, a partir de Hegel podemos mencionar que el proceso dialéctico está constituido por tres elementos: tesis (afirmación de los conceptos, una idea o conocimiento que creemos cierto), antítesis (contradicción de conceptos, 
es la negación, lo opuesto y el ser otro de la tesis) y la síntesis (la unión de la tesis y la antítesis, la unidad), tesis que en algún tiempo tendrá su antítesis y esta a su vez motivara el surgimiento de una nueva síntesis con la cual se buscara llegar nuevamente a la unidad 49,50 .

Por otra parte, Karl Marx, quien fue discípulo de Hegel, mencionó que la dialéctica se encarga de analizar la realidad social y la reoriento al materialismo, en contraposición a las manifestaciones idealistas de Hegel ${ }^{51}$. Marx, asoció la negación de la dialéctica (Antítesis) con el proletario, debido a que el proletariado niega a la burguesía (Tesis), en otras palabras: el proletariado va a pelear contra el orden burgués y lo va a superar creando una sociedad sin clase (Síntesis), en donde no hay más explotación del hombre por el hombre, un comunismo donde las clases habrán sido echadas atrás y se instaurara una sociedad sin explotados ni explotadores ${ }^{52}$.

Por otro lado, Henry Lefebvre (1999) menciona que la dialéctica tiene leyes y éstas deben cumplirse con total cabalidad al implementar este método donde la primera de ellas: a) Ley de la interacción universal: que nos dice que nada existe aisladamente, todo existe en una relación estructural, con todos los demás elementos de la realidad. (b) Ley del movimiento universal: la realidad no está dada de una vez y para siempre está en constante movimiento (c) Ley de la unidad de los contrarios: los elemento de la contradicción a la vez que son opuestos conforman una unidad debido a que se necesitan mutuamente para poder coexistir (Es el elemento antítesis). (d) Ley de la transformación de la cantidad en cualidad: una vez acumulada una cierta cantidad de datos de una misma realidad por la misma dinámica de la dialéctica se produce un cambio cualitativo hacia una realidad nueva. (e) Ley de desarrollo en espiral: Cada nueva situación incluye la anterior ${ }^{53}$.

\section{Articulación hermenéutica dialéctica}

Como consecuencia de todo lo anterior, la combinación entre Hermenéutica y Dialéctica fue inicialmente descrita por Jürgen Habermas en el sentido que desde el punto de vista del pensamiento, sintetiza los procesos comprensivos y críticos en la comunicación entre seres humanos ${ }^{4}$, razón por la cual menciona que es necesario conjuntar las bondades de la hermenéutica y la dialéctica para generar un giro en el 
análisis de datos al no solo comprender, si no llegar hasta una argumentación profunda de los mismos ${ }^{54}$.

Al articular ambos métodos, se cancela todo motivo que no sea la búsqueda cooperativa de la verdad y se argumentan los datos hasta llegar a la certeza de la esencia misma de las $\operatorname{cosas}^{3}$, provocando que el investigador en enfermería descubra semejanzas y contradicciones en la argumentación de los locutores, sin mayor coerción que la del mejor argumento ${ }^{55}$, comprendiendo que nunca será posible tener un conocimiento objetivo del significado de un texto ${ }^{21}$, que nada existe aisladamente, y que la realidad no está dada de una vez y para siempre.

Es así, que podemos concluir, que la hermenéutica y la dialéctica son dos métodos de análisis que todo investigador en enfermería debe emplear durante el análisis cualitativo de datos, debido a que el investigador, como persona humana es un ser interpretativo por naturaleza (Hermenéutica) ${ }^{5,8}$ y busca intensamente la comprensión y significado de los fenómenos a través del razonamiento, la argumentación y el diálogo (Dialéctica) ${ }^{35}$, en el que el personal de enfermería al dialogar e interpretar, debe intentar ponerse en el lugar del autor tal como lo recomienda Dilthey para poder así, revivir el acto creado ${ }^{19}$.

\section{Conclusiones}

La hermenéutica permite llegar a un entendimiento nunca total o completo de los datos $^{20}$, por lo que nada de lo que se interpreta puede ser entendido de una sola vez y para siempre, es un proceso progresivo, en el que la comprensión siempre será un resultado parcial, inacabado, incompleto es un proceso que por las características propias del circulo hermenéutico se prolonga al infinito ${ }^{27}$, por lo cual jamás podremos decir que proporcionamos una interpretación definitiva o tenemos un saber acabado y absoluto 25 .

Mediante la dialéctica se busca llegar a la verdad por medio del diálogo, contraponiendo argumentaciones aparentemente opuestas y frecuentemente complementarias $^{40,41}$, en la que según Hegel, el proceso dialéctico está constituido por una tesis, antítesis y síntesis ${ }^{50}$, en la cual si consideramos las leyes que ofrece Henry Lefebvre, concluiremos que debemos considerar el todo, todo que es cambiante y nada es de una vez y para siempre ${ }^{53}$. 
En síntesis, el emplear la hermenéutica dialéctica como técnica de análisis cualitativo en enfermería, permite interpretar datos sin fragmentación al considerar la condición histórica del interlocutor y agrupar dentro de las narrativas los concesos y disensos del discurso para llegar a una verdad o unidad a partir del diálogo, tomando en cuenta que la conclusión obtenida no es un saber absoluto y por tanto esta propenso a cambios constantes, que mediante el diálogo es posible ajustar, con el único y último objetivo de alcanzar la verdad.

\section{Referencias}

1. Martínez N. La ética y la investigación en enfermería. Rev Cuba en Enfermería. 2010;26(1):18-29.

2. Coello E. Blanco N. Reyes Y. Los paradigmas cuantitativos y cualitativos en el conocimiento de las ciencias médicas con enfoque filosófico-epistemológico. Edumecentro. 2012;4(2):137-146.

3. Lobo M. Ramierez L. Conteras F. Hermenéutica y Dialéctica [Internet]. República Bolivariana de Venezuela. Universidad Pedagógica Experimental Libertador. 2013 [consulta 2017 Feb 23]. Disponible http://maidalobo.blogspot.com.br/2013/03/hermeneutica-dialectica.html

4. De Souza M. La artesania de la investigación cualitativa. Buenos Aires Argentina. Salud Colectiva; 2009. pág.1-332.

5. Morales C. Capítulo I Historia de la hermenéutica [Internet]. Comprender el derecho. 2011 [consulta 2017 Feb 14]; pág. 11-42. Disponible en: http://190.242.62.234:8080/jspui/bitstream/11227/190/3/03 CAPÍTULO I.pdf

6. D’Agostini F. Analíticos y continentales. Catedra, editor. Madrid; 2009. pág. 1-552.

7. Vilallobos LC. Reseña y aportes de la hermenéutica: miradas desde el constructivismo. Rev Psicol. 2012; 21(1):57-84.

8. Marín J. Experiencia y nitud: La hermenéutica escéptica de Odo Marquard. 2016;21(72):27-39.

9. Lleras V, Sepúlveda J, Castillo E. Hermenéutica Jurídica: Legislación Nacional e Internacional. $4^{\circ}$ Version. Medellín Colombia. UNIREMINGTON. 2016. pág. 1-83

10. Picontó T.Teoría general de la interpretación y hermenéutica jurídica: Betti y Gadamer. Dialnet [Internet]. 1992 [consulta 2017 Feb 17];9:223-48. Disponible en: https://webcache.googleusercontent.com/search?q=cache:-

PxG2jTuwNkJ:https://dialnet.unirioja.es/descarga/articulo/142224.pdf+\&cd=4\&hl=es\&ct= clnk\&gl=mx

11. Lince R, Amador J. La hermeneútica filosófica y la interpretación de la obra de arte 
[Internet]. Universidad Nacional Autonoma de Mexico. 2013 [consulta 2017 Feb 15]. pág. 35-90. Disponible en: https://www.researchgate.net/publication/260710914 LA HERMENEUTICA FILOSOFI CA Y LA INTERPRETACION

12. Aguilar L, Hans $O$, Gadamer G. La hermenéutica filosófica de Gadamer. Sinéctica [Internet]. 2004 [consulta 2017 Sep 1];24:61-4. Disponible en: http://www.redalyc.org/pdf/998/99815918009.pdf

13. Reyes G. El problema hermenéutico de la historia efectual en el acercamiento al texto bíblico. Rev Bíblica. 2013;75:181-92.

14. De Santiago E. La hermenéutica metódica de Friedrich Schleiermacher: Otros logros Rev Estud críticos. 2012;3:148-73.

15. Martínez M. Hermenéutica y análisis del discurso como método de investigación social. Paradigma [Internet]. 2002;23:1-13.

16. Schleiermacher FDE. Los discursos sobre hermeneutica.

17. Fayad F. Hermenéutica: el aporte alemán a la interpretación de la ley. Dixi. 2013;15(18):133-6.

18. Rivas Y, Briceño J. La hermenéutica: Sus orígenes, evolución y lo que representa en este convulsionado periodo. Rev Academia. 2012;11(23):225-33.

19. Da Trinidade Y. López Y. La Hermenéutica en el pensamiento de Wilhelm Dilthey. Griot Rev filos. 2015;11(2):324-41.

20. Ciurana E. Antropologia Hermeneutica (Sobre Dilthey; Heidegger y Gadamer). Universidad del Salvador Vicerrectorado de Investigación y Desarrollo [Internet]. 2001 [consulta 2017 Aug 6]. Disponible en: http://www.iecomplex.com.br/textos/herm.htm

21. Rueda P, Vilarroel I. El método Hermenéutico-Dialectico una estrategia para las ciencias de la conducta. 2015 [consulta 2017 Feb 14]; Disponible en: https://documents.mx/documents/el-metodo-hermeneutico-dialectico.html

22. Osorio F, Martínez M, Toledo U. et al. Epistemología y ciencias sociales: ensayos latinoamericanos [Internet]. Santiago de Chile. LOM ediciones. pág 1-334. 2014 [consulta 2017 Sep 4 ] Disponible https://www.researchgate.net/publication/261025688 Epistemologia y ciencias sociale s ensayos latinoamericanos

23. León E. El giro hermenéutico de la fenomenológica en Martín Heidegger. Rev Latinoam. 2009;(22):1-15.

24. De La Maza L. Fundamentos de la filosofía hermenéutica: Heidegger y Gadamer. Teol y Vida. 2005;46:122-38. https://doi.org/10.4067/S0049-34492005000100006

25. Minayo M. Los conceptos estructurantes de la investigación cualitativa. Salud Colectiva. 2010;6(3):251-61. https://doi.org/10.18294/sc.2010.283 
26. Medina J. El proceso de comprensión en el análisis de datos cualitativos en educación. Magis Rev Int Investig en Educ. 2014;7(14):39-54. https://doi.org/10.11144/Javeriana.M714.EPCA

27. González J, Coquies U. Las cooperativas y sus prácticas de resolución de conflictos desde el enfoque cualitativo. Rev Latinoam Metodol la Investig Soc. 2011;2:44-58.

28. Carrera M. Inciarte A. Marín F. Sistema metodológico de investigación desde la perspectiva crítica de diversos actores sociales. Multiciencias. 2012;12:81-7.

29. García J. El debate Gadamer-Habermas: Interpretar o transformar el mundo. Redalyc Contrib desde Coatepec. 2006;10:11-21.

30. Habermas J. Rorty R. Vattimo G. et al. El ser que puede ser comprendido es lenguaje: Homenaje a H-G Gadamer. España. SINTESIS. 2001. pág. 1-14.

31. Rivero P. Cuestiones hermenéuticas de Nietzche a Gadamer. México. UNAM, Facultad de Filosofía y Letras, Posgrado de Maestría y Doctorado. ITACA. 2006. pág. 1-191.

32. González M. De la técnica a la hermenéutica. Rev del Col Filos. 2000;11:119-26.

33. Abellán Á. De la Dialéctica a la Dialógica. Rev del humanismo español e hiberoamericano. 2012;31:97-125.

34. Marx K. Dialéctica. Historia de la filosofia. Volumen 3: Filosofia Contemporanea. Editorial Edinumen.

35. Reyes C. La mayéutica de Sócrates en la formación humana. Planeac y evaluación Educ. 2008;43:3-10.

36. González R. Pérez L. Pierre Hadot: El cuidado de sí y la mayéutica socrática como ejercicio espiritual. Cienc ergo sum. 2016;23(1):26-34.

37. Abbagnano. Paci. Viano. et al. La Evolucion de la Dialéctica. Martínez Roca, editor. 1971. pág.1-275.

38. Varas J. Sócrates y Mayéutica. Obstet y Ginecol. 2009;4(1):7-8.

39. Chacón Á. Covarrubias F. El sustrato platónico de las teorías pedagógicas. Tiempo de Educar. 2012;13(25):139-59.

40. Cano M. Modelo epistemológico de la teoría tridimensional del derecho. Convergencia. Rev de Ciencias Sociales. 2011;18(57):209-28.

41. Cañas R. La Dialéctica en la filosofía griega. Rev las Sedes Reg [Internet]. 2010;22(11):37-56.

42. Abbagnano N. Diccionario de filosofia. 4th ed. México: Fondo de cultura económica; 2004. pág. 295-303.

43. Dussel E. Métodos para una filosofía de la liberación. Sigueme. editor. España; 1974 17-31 p.

44. Citio Z., Quíos A, Apolófanes, Calcedonia H, Heraclea D, Citio P, Cleantes E. Los estoicos antiguos. Gredos, editor. Madrid; 1996. 1-334 p. 
45. Muñoz E. Los estoicos antiguos. Rev Filos la Univ Costa Rica. 1993;(Extra 75-76):10518.

46. Martínez H. Kant: Una ética para la modernidad. Diálogos de Saberes. 2006;(24):18194.

47. Horstmann R. La contradicción en Hegel. Rev Latinoam Filos. 2009;2(35):189-206.

48. Wilson E. El mito de la dialectica. [consulta 2017 Sep 6];215-37. Disponible en: http://humanidad.webcindario.com/materiales/wilson-el-mito-de-la-dialectica.pdf

49. Martinez A. La dialectica hegeliana - YouTube [Internet]. 2016 [consulta 2017 Feb 20]. Disponible en: https://www.youtube.com/watch?v=PoZQPHrCZnU

50. Fiscer G. Friedrich Engels y el materialismo histórico. Rev Clases historia. 2012;326:133.

51. Bruno D. La dialéctica histórica de Karl Marx: aproximaciones metodológicas para una teoría del colapso capitalista. Cons Latinoam ciencias Soc. 2011;1:75-86.

52. Botica P. Dialéctica en Marx [Internet]. 2016 [consulta 2017 Feb 20]. Disponible en: https://www.youtube.com/watch?v=G9PhNpZzfN0

53. Lefebvre R.Traducción De Benitez E. Lógica formal Lógica dialéctica. Primera ed. Anthropos P, editor. España; 1970. 349 p.

54. Hoyos G. Hermenéutica Práctica: Habermas VS. Gadamer. Fac artes y humanidades [Internet]. 2003 [consulta 2017 Sep 1];1-17. Disponible en: http://www.pedagogica.edu.co/storage/folios/articulos/folios17 06arti.pdf

55. Malo E. Mejor argumento, acción comunicativa yclases sociales. Una crítica de lapropuesta de Habermas. Athenea Digit Rev Pensam e Investig Soc. 2005;(7):16-39. 